\title{
End Effect in Uniaxial Compression Test of Marble
}

\author{
Wu Zhichou ${ }^{1,}$,, Zhang Ning ${ }^{1}$, Wang Jiabo ${ }^{2}$, , Wang Shuo ${ }^{2}$ \\ ${ }^{1}$ School of Science, Nanjing University of Science and Technology, Nanjing, China \\ ${ }^{2}$ National Defense Engineering Institute, Army Engineering University, Nanjing, China
}

\section{Email address:}

497052393@qq.com (Wu Zhichou),wjb412723@163.com (Wang Jiabo)

${ }^{*}$ Corresponding author

\section{To cite this article:}

Wu Zhichou, Zhang Ning, Wang Jiabo, Wang Shuo. End Effect in Uniaxial Compression Test of Marble. Science Discovery. Vol. 7, No. 6, 2019, pp. 415-418. doi: 10.11648/j.sd.20190706.17

Received: November 15, 2019; Accepted: December 3, 2019; Published: December 9, 2019

\begin{abstract}
In order to reveal the influence of friction between loading plate and test piece on test strength, Alloy steel, polytetrafluoroethylene and rubber are used as cushion, Uniaxial compression tests of marble cubes under different end contact conditions were carried out. The test results show that, When the cushion is thick, the greater the cushion stiffness is, the greater the test strength is; when the deformation modulus of cushion material is significantly smaller than the tested piece, the greater the cushion thickness is, the lower the test strength is,so the characteristics of cushion have significant influence on the test strength and failure characteristics. Based on the transverse deformation capacity of loading plate and specimen, the influence rule of different end conditions on test strength is given. The comparative test is carried out with a cylinder sample with a height diameter ratio of 2.0, Based on the viewpoint of relative strength, a new idea of reducing friction in rock test is put forward.
\end{abstract}

Keywords: Marble, Uniaxial Compression, Testing Intensity, Friction Effect

\section{大理岩单轴压缩试验的端部效应}

吴志酬 ${ }^{*}$, 张宁 ${ }^{1}$, 王贾博 ${ }^{*}$, 王硕 ${ }^{2}$

1南京理工大学理学院, 南京, 中国

2陆军工程大学国防工程学院, 南京, 中国

\section{邮箱}

497052393@qq.com（吴志酬）,wjb412723@163.com（王贾博）

摘要: 为揭示加载板与试件之间摩擦对测试强度的影响, 采用合金钢、聚四氟乙烯、橡胶 3 种材料作为垫层, 开展了不 同端部接触条件下大理岩立方体的单轴压缩试验。试验结果表明, 垫层较厚时, 垫层刚度越大, 测试强度越大; 当垫 层材料变形模量显著小于被测试件时, 则垫层厚度越大, 测试强度越低, 可见垫层特性对测试强度和破坏特征具有显 著影响。基于加载板和试件的横向变形能力给出了不同端部条件对测试强度的影响规律。采用高径比为 2.0 的圆柱试样 开展对比试验, 基于相对强度的观点提出了岩石试验减摩新思路。

关键词: 大理岩, 单轴压缩, 测试强度, 摩擦效应 


\section{1. 引言}

对摩擦效应的研究一直是岩石力学试验领域的热点 和难点。以往的研究表明, 在开展双向受压和真三轴试验 时, 端部的摩擦效应可以对试验结果产生严重的不利影响。 尤明庆等[1]通过大理岩的单轴压缩试验发现, 端部加聚四 氟乙烯垫片可以显著降低试件的的单轴抗压强度。范鹏贤 等[2-4]对红砂岩开展的单轴压缩和双向压缩试验表明, 加 载板和试件之间的摩擦可以显著的影响被测试件的强度 特性。杨圣奇等[5]通过大理岩的单轴压缩实验得出, 岩石 强度的尺寸效应是端面摩擦效应作用造成岩石内部应力 分均匀分布的缘故。潘鹏志[6]等通过对单轴压缩破裂过程 进行模拟, 表明试样端部的摩擦情况对岩石变形和强度特 性影响较大。麦戈等[7]通过对岩石单轴压缩强度的端部效 应进行了数值仿真实验, 发现随着摩擦系数的增加, 岩石 试件表现出来的单轴抗压强度趋于增大。郭保华等[8]采用 FLAC程序分析了圆柱岩样单轴压缩的摩擦效应, 得出端 部固定岩样强度值均大于端部光滑岩样, 反应出端部摩擦 的影响。

数十年来, 很多学者通过理论建模, 数值模拟[9-11]、 对比试验[12-14]等试图量化其影响, 然而迄今为止, 尚未 有很好的办法解决这一难题。一般采取减摩措施来降低端 部摩擦效应, 如 K. Mogi[15] 总结了几种减小端部效应的 方法, 并指出采用不同的润滑剂所得的结果差别较大。但 目前的试验机仍以润滑减摩为主[16]。对于另一种常用的 使用垫片的减摩方法, 目前还以定性描述为主, 缺乏足够 的实证研究。

\section{2. 试验方法}

为了定量评估端部条件的影响, 开展了大理岩在不同 垫层条件下的单轴压缩试验。试件尺寸近似呈立方体，棱 长99 101 mm。立方体试件强度测试通过电液伺服万能试 验机完成。试验步骤为: 对试件进行篎选和随机分组, 然 后在上下表面垫上特定材质和厚度的垫片, 置于万能试验 机上下压盘之间, 对中调平, 采用 $0.2 \mathrm{~mm} / \mathrm{min}$ 的速度加载 直至试件破坏。试件的强度通过峰值力和实际截面积计算。

试验采用合金钢、聚四氟乙烯和橡胶3种不同性质的 端部接触材料, 对于常被用作减摩垫层的聚四氟乙烯, 设 置了 $0.3 \mathrm{~mm} 、 1 \mathrm{~mm} 、 2 \mathrm{~mm} 、 5 \mathrm{~mm} 、 10 \mathrm{~mm}$ 等 5 种不同厚 度，每种工况不少于 4 个有效试件。

\section{3. 主要试验结果}

\section{1. 测试强度}

主要试验结果见表1。

从表 1 中可以看出, 端部接触工况对大理岩试件的测 试强度有明显的影响。直接加载时的测试强度最高, 是最 低测试强度（垫 $10 \mathrm{~mm}$ 聚四氟乙烯）的 4.00 倍。同样采用 聚四氟乙烯垫层, 厚度为 $0.3 \mathrm{~mm}$ 时, 测试强度最大, 是厚 度 $10 \mathrm{~mm}$ 工况时的 3.08 倍。端部对试件变形约束较强时, 试件的测试强度较大; 当垫层变形刚度较小时, 试件的测
试强度则随垫层厚度的增大而减小。由此可见，目前的试 验方法中存在较强的端部效应, 易导致强度测试结果的失 真。

表1 主要测试结果。

\begin{tabular}{llll}
\hline 工况 & & 测试强度参数 \\
\hline 垫板材质 & 厚度 $(\mathrm{mm})$ & 均值(MPa) & 标准差(MPa) \\
合金钢 & $/$ & 79.76 & 8.92 \\
& 0.3 & 61.48 & 4.00 \\
聚四氟 & 1 & 52.67 & 5.25 \\
乙烯 & 2 & 46.79 & 7.16 \\
& 5 & 32.58 & 7.61 \\
橡胶 & 10 & 19.95 & 1.82 \\
\hline
\end{tabular}

\section{2. 破坏模式}

试验结果表明, 不同工况下试件的破坏模式相差很大。 垫层较厚时, 若垫层刚度较小 (如橡胶垫层、 $10 \mathrm{~mm}$ 聚四 氟乙烯垫层），试件多发生䢃裂破坏，破坏过程较温和， 破坏后试件尚有一定的承载能力; 若垫层刚度较大, 试件 多发生剪切破坏, 破坏较剧烈, 破坏时伴随较大响声, 破 坏后试件不能保持完整, 基本没有残余承载力。不同垫层 的典型破坏模式见图1。

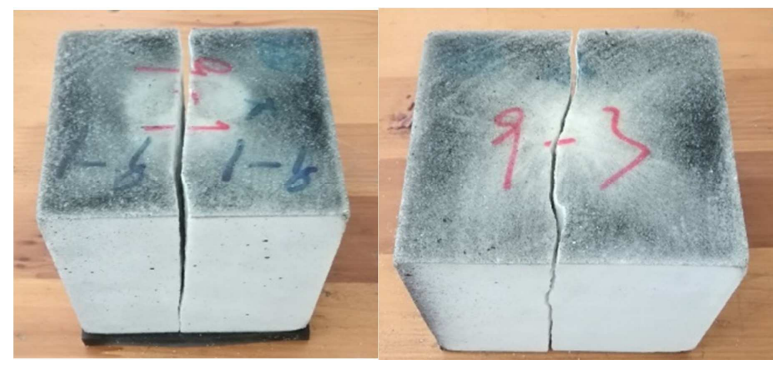

(a) 单裂纹拉裂 ( $10 \mathrm{~mm}$ 聚四氟乙烯板)

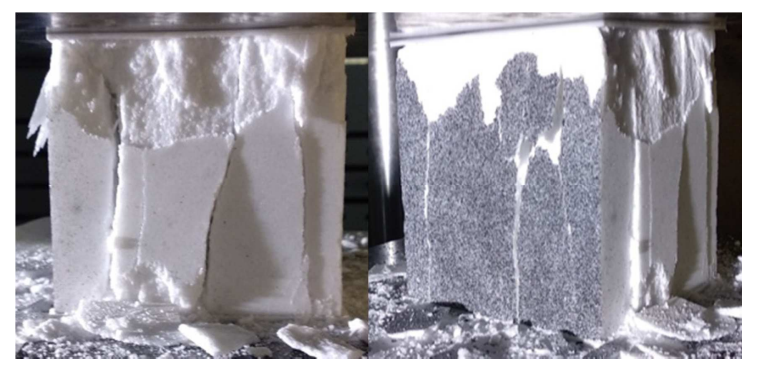

(b) 多组拉伸裂隙䢃裂（1mm、2mm聚四氟乙烯板）

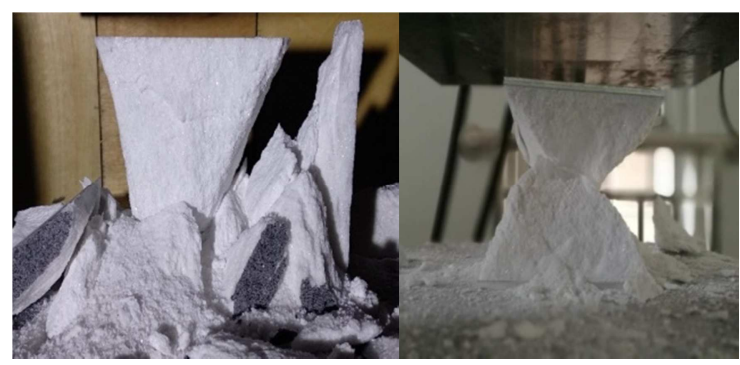

(c) $\mathrm{X}$ 型或雉形剪切破坏（直接加载）

图1 典型破坏模式。 
垫层采用聚四氟乙烯时, 垫层较薄 $(0.3 \mathrm{~mm})$ 时, 试件在峰值荷载时发生剧烈破坏, 多发生 X 型或雉形剪 切破坏。随着垫层厚度增加, 破坏剧烈程度逐渐降低, 破坏模式逐渐由剪切剧烈破坏转变为张剪复合破坏和 䢃裂破坏。采用较厚的软垫层时, 往往仅产生一两条与 加载方向平行的主裂纹。试件破坏模式与测试强度之间 具有较强的关联性, 发生剧烈的X型剪切破坏模式时测 得的强度往往最高, 张剪复合破坏或䢃裂剥离破坏时强 度中等, 多裂纹䢃裂破坏时强度较低, 而单裂纹䢃裂对 应的强度最低。

破坏模式反映了加载端对试件变形的约束。垫层变 形能力弱时, 加载端限制了试件的侧向变形, 形成环筷 效应, 使裂纹难以纵向发展, 因此形成X型或雉形破坏。 而垫层刚度小, 加载时垫层侧向变形大, 形成离心的侧 向张力, 易引起䢃裂破坏, 并导致试件在较低应力下破 坏。

\section{4. 讨论}

为定量评估摩擦效应, 采用相同的材料制备了 10 件 高径比为 2.0 的圆柱形标准试件。由于试件高径比较大, 且采取了一定的减摩措施, 因此摩擦效应基本可以忽略, 其测试强度接近材料本身的强度。经测试, 该大理岩试 样有效测试强度均值为 $51.52 \mathrm{MPa}$, 标准差为 $2.12 \mathrm{MPa}$, 试件变形弹性段的变形模量约 $40.4 \mathrm{GPa}$, 荷载为峰值强 度一半时的泊松比约为 0.15 , 接近峰值时的泊松比约 为 0.38 。

定义两个无量纲参数: 等效横向变形模量和相对强度。 等效侧向变形模量反应端部和试件的相对侧向变形能力, 定义为在轴向力作用下材料产生单位横向应变所需的应 力, 其衍生出归一化横向变形模量, 即相应材料的等效横 向变形模量与被测红砂岩等效横向变形模量的比值。相对 强度则定义为测试强度与标准试件测试强度的比值。经过 归一化处理, 不同工况下的归一化横向变形模量及相对强 度见图2。

从图 2中可以看出, 被测试件的相对强度和垫层的归 一化横向变形模量和正相关。当与被测试件直接接触部分 的横向变形能力较弱时, 摩擦约束会提高试件的测试强度, 反之则会降低被测试件的测试强度。

不同厚度的聚四氟乙烯垫片得到的测试强度相差很 大。聚四氟乙烯垫片厚度与相对强度之间的关系见图 3。 图3中可以看出, 聚四氟乙烯垫片很薄时, 试件测试强度 虽小于直接加载工况, 但相对强度仍显著大于 1 。随着垫 片厚度的增加, 相对逐渐下降, 垫片厚度超过 $1 \mathrm{~mm}$ 时, 相对强度趋近于 1 , 即与标准试件强度相当, 垫片厚度进 一步增加时, 相对强度单调降低。

结合本文的试验结果, 试验机压板直接压缩试样时, 由于合金钢的侧向变形刚度远大于被测试件, 摩擦效应能 提供向心的摩擦约束, 试样的侧向膨胀受到抑制, 可以显 著提高试件的测试强度; 采用柔性垫层时, 垫层会产生较 大的侧向膨胀, 产生指向外缘的摩擦力, 倾向于降低被测 试件的强度。

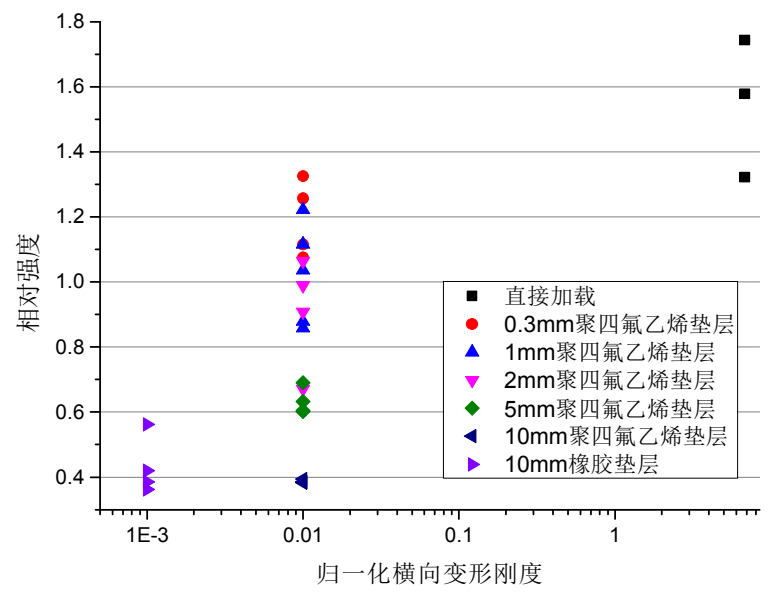

图2 归一化后的测试结果。

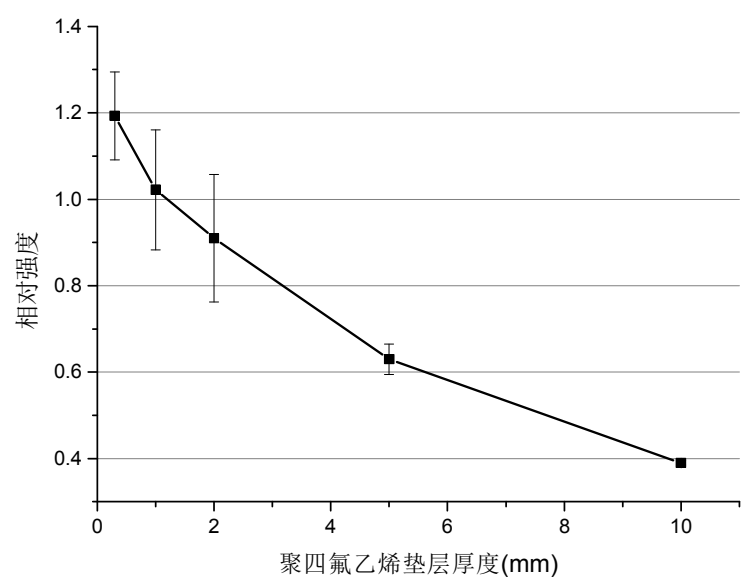

图3 聚四氟乙烯垫片厚度与相对强度的关系。

聚四氟乙烯的减摩作用并不是由其相对较低的摩擦 系数导致的, 而更多的应归结为其柔性变形不同程度地匹 配了试件和加载端的侧向位移。不同厚度聚四氟乙烯垫片 起到的减摩效果不同。以大理岩单轴压缩试验为例, 采用 $0.3 \mathrm{~mm}$ 聚四氟乙烯垫片, 虽然相对强度由 直接加载的 1.55 降低至 1.19 , 但仍然较标准试件的测试结果显著偏大, 而采用 $1 \mathrm{~mm}$ 厚度的聚四氟乙烯垫片得到测试结果则接 近真实强度的（两者比值约 1.02）。因此, 从横向变形 匹配的角度合理选择垫片的厚度, 可以有效减小甚至消除 摩擦效应, 使试验结果接近被测试件的真实响应。

相对于单轴受压情况，双向受压、真三轴或卸载应力 路径下, 被测试件的受力状态更复杂。采用横向变形匹配 的垫片相较于采用润滑剂减小摩擦因数更加简便可靠, 但 垫层材质、厚度需要根据被测试件的物理力学性能合理选 择。通过试验对垫层和试件间横向变形匹配关系进行深入 研究仍然是揭示真实摩擦效应并合理评估其影响的最优 选择。

\section{5. 结论}

针对端部摩擦效应问题, 采用大理岩试件开展了单轴 压缩试验, 试验发现如下: 
垫层横向变形特性对测试强度有显著影响, 相同几何 形状和材质的被测试件的测试强度可相差3倍以上, 必须 合理评估端部接触条件对测试结果的影响;

刚度较大的端部约束倾向于提高被测试件的测试强 度, 而软弱垫层的摩擦效应则与厚度密切相关。厚度一致 时, 垫层的刚度越大, 被测试件的测试强度越大, 垫层材 质相同且横向变形模量较小时, 垫层厚度越小, 被测试件 的测试强度越大。

结合标准试件测试结果, 给出了不同工况下归一化横 向变形模量与相对强度的关系, 垫层横向变形能力由弱到 强变化时, 对被测试件的摩擦作用逐步由约束过渡到扩张, 只有端部与被测试件的横向变形能力基本匹配时, 测试结 果才能代表材料的真实强度。对于大理岩试件, 采用 $1 \mathrm{~mm}$ 厚度的聚四氟乙烯垫片开展单轴压缩试验能够基本反应 被测试件的强度特征。

提出从横向变形匹配的角度合理选择垫片的减摩方 法, 为提高立方体压缩、真三轴加卸载等试验的可靠性提 供了新的思路。

\section{参考文献}

[1] 尤明庆. 端部效应对岩石真三轴压缩强度的影响 [J]. 岩石 力学与工程学报, 2016, 35(增1): 2 603-2 607.

[2] 范鹏贤, 李䅎, 赵跃堂, 等. 茂木式真三轴试验机的端部 摩擦效应 [J]. 岩石力学与工程学报, 2017, 36(11): 2720-2730.

[3] 范鹏贤, 李颖, 赵跃堂, 等. 红砂岩卸载破坏强度特征试 验研究, 岩石力学与工程学报, 2018, 37(4): 852-861.

[4] 范鹏贤, 王贾博, 刘家贵, 等. 端部条件对红砂岩试件测 试强度影响的试验研究, 应用基础与工程科学学报, 2019, 录用待刊。

[5] 杨圣奇, 苏承东, 徐卫亚. 岩石材料尺寸效应的试验和理 论研究 [J]. 工程力学, 2005, 22(4): 112-118.(YANG Shengqi, SU Chengdong, XU Weiya. Experimental and theoretical study of size effect of rock material [J]. Engineering Mechanics, 2005, 22 (4): 112-118. (in Chinese))
[6] 潘鹏志, 周辉, 冯夏庭.加载条件对不同尺寸岩石单轴压缩 破裂过程的影响研究 [J].岩石力学与工程学报, 2008, 27(增 2).

[7] 麦戈等.岩石单轴压缩端部效应的数值仿真分析[J]. 长江科 学院院报,2013,30(6):68-71.

[8] 郭保华. 岩样尺度、孔道及端部摩擦效应的数值分析 [J]. 岩 石力学与工程学报,2009,28（增2）:3391-3400.

[9] 石 露, 李小春. 真三轴试验中的端部摩擦效应分析 [J]. 岩 土力学, 2009, 30(4): 1 159-1 164.(SHI Lu, LI Xiaochun. Analysis of end friction effect in true triaxial test[J].Rock and Soil Mechanics, 2009, 30(4): 1 159-1 164.(in Chinese))

[10] XU YH, CAI M, ZHANG XW, et al. Influence of end effect on rock strength in true triaxial compression test [J]. Canadian Geotechnical Journal, 2017, 54 (6): 862-880.

[11] 何浩宇, 石露, 李小春, 等. 基于新型茂木式试验机的真三 轴试验及加载边界效应研究 [J]. 岩石力学与工程学报, 2015, 34(增1): 2 837-2 844. (HE Haoyu, SHI Lu, LI Xiaochun, et al. True triaxial tests with new mogi-type true triaxialtest apparatus and its loading boundary effect [J]. Chinese Journal of Rock Mechanics and Engineering, 2015, 34 (Supp.1): 2 837-2844. (in Chinese))

[12] 尤明庆, 苏承东, 徐 涛. 岩石试样的加载卸载过程及杨氏 模量 [J]. 岩土工程学报, 2001，23(5)：588-592. (YOU Mingqing, SU Chengdong, XU Tao. Loading or unloading process in axial direction and Young $\mathrm{s}$ modulus of rock specimen [J]. Chinese Journal of Geotechnical Engineering, 2001, 23 (5): 588-592. (in Chinese))

[13] Xia-Ting Feng, Xiwei Zhang, Chengxiang Yang, et al. Evaluation and reduction of the end friction effect in true triaxial tests on hard rocks [J]. International Journal of Rock Mechanics \& Mining Sciences. 2017, 97: 144-148.

[14] 张希巍, 冯夏庭, 孔瑞, 等. 硬岩应力-应变曲线真三轴仪 研制关键技术研究 [J]. 岩石力学与工程学报, 2017, 36(11): 2629-2640.

[15] MOGI K. Experimental rock mechanics [M]. London: Taylor and Francis, 2006.

[16] Haimson B, Rudnicki JW. The effect of the intermediate principal stress on fault formation and fault angle in siltstone. Journal of Structural Geology. 2010, 32: 1701-1711. 